\title{
Embodiment and learning of abstract concepts (such as algebraic topology and regression to the mean)
}

\author{
Arthur M. Glenberg ${ }^{1,2,3}$ (D) \\ Published online: 1 September 2021 \\ (c) The Author(s), under exclusive licence to Springer-Verlag GmbH Germany, part of Springer Nature 2021
}

\begin{abstract}
This video is a proof of concept that ideas from embodied cognition can be used to understand how the brain and cognitive systems deal with very abstract concepts. The video teaches regression to the mean using three ideas. The first idea is directly related to embodied cognition: abstract concepts are grounded in perceptual, motor, and emotional systems by using successive levels of grounding within an extended procedure. The second idea is that this sort of grounding often requires formal instruction: a teacher needs to develop the sequence in which the concepts are grounded and the methods of grounding. That is, at least some abstract concepts are unlikely to be learned through an individual's unstructured interactions with the world. The third idea is that humans are hyper-social, thus making formal instruction possible. To the extent that the viewer learns the abstract concept of regression to the mean, then the video demonstrates how an embodied theory of abstract concepts could work.
\end{abstract}

Keywords Abstract concepts · Embodied cognition · Grounding $\cdot$ Regression to the mean

This contribution to the special issue on "Concrete constraints on abstract concepts" consists primarily of an online video illustrating how concepts from embodied cognition can be used to teach an abstract concept. The video may be accessed through the supplementary materials. Another article in the special issue, Fischer et al. (2021), contextualizes the video by discussing its relation to the literature on embodied mathematical cognition.

Supplementary Information The online version contains supplementary material available at https://doi.org/10.1007/s00426-021-01576-5.

Acknowledgements I thank Friedemann Pulvermüller for organizing the workshop on the Neurocognition of Abstract Concepts at the Berlin School of Mind and Brain and Michael Arbib for issuing the challenge that led to this video. I also thank Robert Ewing for his time and skill in taping and editing the lecture.

\section{Reference}

Fischer, M.H., Glenberg, A.M., Moeller, K., \& Shaki, S. (2021). Grounding (fairly) complex numerical knowledge: An educational example. Psychological Research. https://doi.org/10.1007/ s00426-021-01577-4

Publisher's Note Springer Nature remains neutral with regard to jurisdictional claims in published maps and institutional affiliations.
Arthur M. Glenberg

glenberg@asu.edu

1 Department of Psychology, Arizona State University, Tempe, AZ 85287-1104, USA

2 Department of Psychology, University of Wisconsin-Madison, Madison, WI, USA

3 INICO, Universidad de Salamanca, Salamanca, Spain 